\author{
В. И. Голик (D), О.Г. Бурдзиева $(\mathbb{D}$, Б. В. Дзеранов \\ Геофизический институт Владикавказского научного центра \\ Российской академии наук, Россия, 362002, РСО-Алания, г. Владикавказ, \\ ул. Маркова, 93a, e-mail: v. i. golik@ mail. ru
}

Статья поступила: 20.03.2021, доработана: 20.04.2021, принята к публикации: 11.05.2021

Резюме: Актуальность работы. Рассмотрены актуальные вопросы рационализации природопользования, включающие в себя установление величины и закономерностей экологического риска технологических процессов, оценку процессов коммуникации техногенных продуктов и прогнозирование экологической зависимости биоты от горного производства при подземной разработке месторождений Садонского рудного узла. Целью исследований является определение форм взаимодействия природных и технических систем, определяющих параметры геомеханики участка земной коры в районе освоения недр путем исследования напряженно-деформированного состояния массивов для разработки приемлемых вариантов добычи металлических руд. Методы исследования. Приведены результаты моделирования состояния рудовмещающих массивов на оптически активных материалах. Предложен коэффицциент надежности для оценки безопасности горных технологий по развитию критических напряжений и деформаций. Результаты работы. Установлена адекватная зависимость между характером силового воздействия на природные и искусственные массивы и их устойчивостью с корреляцией между напряженностью массива и сохранностью земной поверхности, как гаранта безопасности экосистем биосфреры. Даны результаты моделирования напряженности массива методом конечных элементов, распределения давления по контуру выработки на крепь и исследования фильтрационной неоднородности руд. Обобщены результаты анализа ретро- и перспектив развития способов управления состоянием массивов при разработке месторождений. Сделан вывод о возможности обеспечения оптимального соотношения напряжений в элементах геомеханической системы геофизическими методами в рамках мониторинга состояния горного массива и подземных выработок. В совокупности рекомендации представляют собой программу выхода горных предприятий региона из затяжного кризиса и восстановления былого потенциала одного из флагманов цветной металлургии, реализация которой фрормирует экономический, экологический и социальный эффект в условиях депрессивного промышленного региона.

Ключевые слова: Подземная разработка, месторождение, Садонский рудный узел, геомеханика, моделирование, горная технология, напряжения и десормации, геофизические методы, мониторинг.

Для цитирования: Голик В. И., Бурдзиева О.Г., Дзеранов Б. В. Геофизический мониторинг разработки напряженно-деформированных рудовмещающих массивов. Геология и геофизика Юга России. 2021. 11 (2): 63 - 73. DOI: 10.46698/NNC. 2021.57.57.005. 


\title{
Geophysical monitoring of the development of stress-deformed ore massives
}

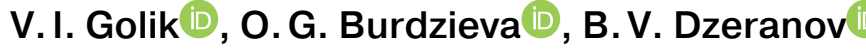 \\ Geophysical Institute of the Vladikavkaz Scientific Centre of the Russian Academy of Sciences, \\ 93a Markova Str., Vladikavkaz 362002, Russian Federation, e-mail: v. i. golik@ mail. ru \\ Reseived: 20.03.2021, revised: 20.04.2021, accepted: 11.05.2021
}

\begin{abstract}
Relevance. Topical issues of rationalization of nature management are considered, including the establishment of the magnitude and regularities of the ecological risk of technological processes, the assessment of the communication processes of technogenic products and the prediction of the ecological dependence of biota on mining during the underground development of the deposits of the Sadonsky ore cluster. The Aim of the research is to comprehensively study the interaction of natural and technical systems that ensure the geomechanical balance of massifs in the area of subsoil development by systematic monitoring of the stressstrain state of the massifs using the methods of complex generalization, analysis of practical experience and scientific achievements in the field of underground ore mining in the massifs. Complex structure, theory and practice of destruction of solid media by means of continuum mechanics and mathematical statistics with the development of economically and environmentally acceptable options for the extraction of metal ores. Methods. The results of modeling the state of ore-bearing massifs on optically active materials are presented. A reliability factor is proposed for assessing the safety of mining technologies for the development of critical stresses and deformations. Results. An adequate relationship has been established between the nature of the force impact on natural and artificial massifs and their stability with the correlation between the strength of the massif and the safety of the earth's surface, as a guarantor of the safety of biosphere ecosystems. The results of modeling the tension of the massif by the finite element method, the distribution of pressure along the contour of the excavation on the support and the study of the filtration heterogeneity of ores crushed for leaching purposes according to the propagation velocity of stress waves are given. The results of the analysis of retro- and prospects for the development of methods for managing the state of rock mass in the development of fields are generalized. A conclusion is made about the possibility of ensuring the optimal stress ratio in the elements of the geomechanical system by geophysical methods within the framework of monitoring the state of the rock mass and underground workings. Taken together, the recommendations represent a program for the recovery of the region's mining enterprises from a protracted crisis and the restoration of the former potential of one of the flagships of nonferrous metallurgy, the implementation of which creates an economic, environmental and social effect in a depressed industrial region.
\end{abstract}

Keywords: Underground mining, deposit, Sadonsky ore cluster, geomechanics, modeling, mining technology, stresses and deformations, geophysical methods, monitoring.

For citation: Golik V. I., Burdzieva 0. G., Dzeranov B. V. Geophysical monitoring of the development of stressdeformed ore massives. Geologiya I Geofizika Yuga Rossii = Geology and Geophysics of Russian South. (in Russ.). 2021. 11 (2): 63 - 73. DOI: 10.46698/VNC. 2021.57.57.005.

\section{Введение}

Горнодобывающая отрасль, являясь лидером среди промышленных технологий по нарушению сплошности земной коры, создает условия для разрушения земной поверхности, изменения форм рельефа и качества флоры и фауны [Каурин и др., 2018; Burdzieva, 2016]. 
Проблема рационализации природопользования включает в себя установление величины и закономерностей экологического риска, оценку процессов коммуникации техногенных продуктов и прогнозирование экологической зависимости окружающей среды от агрессии горного производства [Дребенштедт и др., 2018; Клюев и др., 2020].

Повышенная опасность добычных процессов определяется синергетическим характером проявления, когда развитие отдельных процессов приводит к возникновению других процессов с большим суммарным эффектом, чем простая сумма эффектов.

Процессы освоения запасов обладают синергетическим характером проявления, когда развитие отдельных процессов генерирует нередко и неизвестные процессы с большим суммарным отрицательным эффектом, чем ожидаемая сумма эффектов.

На территории металлических рудников накоплены миллионы тонн хвостов, которые стали реактором химических процессов заражения окружающей среды регионов Северного Кавказа, Юга России и Каспия.

Горные работы сопровождаются образованием техногенных пустот, которые участвуют в перераспределении напряженно-деформированногого состояния массивов.

Успех разработки месторождений полезных ископаемых определяется полнотой учета и интерпретации поведения природных и технических систем, участвующих в развитии напряженно-деформированного состояния природных и искусственных массивов в [Дребенштедт и др., 2018; Рыльникова и др., 2017].

Поэтому исследование механизма возникновения и перераспределения напряжений в массиве горных пород [Дребенштедт и др., 2018; Aksenov et al., 2019] с целью совершенствования технологий разработки напряженно-деформированных рудовмещающих массивов представляет собой крупную научно-производственную проблему.

\section{Методы исследования}

Моделирование состояния массива на оптически активных материалах. Моделирование напряженности массива методом конечных элементов. Характер и закономерности распределения горного давления на жесткую арочную крепь. Измерение напряжений в крепи с помощью датчиков давления. Определение границ зоны пластических деформаций и разрушение пород прозвучиванием массива в скважинах. Измерение фильтрационной неоднородности руд по скорости волн напряжений. Инженерное прогнозирование перспектив совершенствования технологий.

\section{Результаты исследования}

Основой безопасной разработки месторождений полезных ископаемых является профилактика критических напряжений на всех этапах существования.

Состояние массива моделировалось на оптически активных материалах в условиях Садонских массивов, в том числе:

- боковой распор $0,5,1,0,1,5$;

- угол наклона силового вектора к вертикальной оси $\alpha=0$;

- модуль закладки $\mathrm{E}=0,1 \mathrm{MПа;}$

- модуль вмещающих пород - 1,4 МПа. 




Рис. 1. Поля изохром при коэффициенте бокового распора 1,0: слева-открытая камера; справа - заложенная камера I

Fig. 1. Fields of isochrome with a coefficient of side thrust 1.0: on the left-an open chamber; on the right - a filled chamber

Определено, что наиболее напряжена потолочина камер. При вариантах без закладки в междукамерных целиках концентрация напряжений близка к критической (рис. 1).

При необходимости сохранения массивов и земной поверхности выработанное пространство закладывается твердеющими смесями.

Механизмом регулирования геомеханических условий является применение технологий с заполнением пустот твердеющими смесями и хвостами подземного выщелачивания руд.

Степень безопасности горных технологий оценивается коэффициентом надежности $\mathrm{K}_{1}$ :

$$
K_{1}=f\left(V_{o}-V_{3} K_{T}\right),
$$

где $V_{o}$ - объем образованных в массиве пустот, $\mathrm{M}^{3}$;

$V_{3}$ - объем заполненных пустот, м $^{3}$;

$K_{T}$ - доля заполнителя, \%.

Корреляция между уровнем напряжений в массивах и состоянием земной поверхности над ними устанавливается исследованиями с применением методов шахтной геофизики.

Полученные результаты систематических исследований свидетельствуют об адекватной зависимости между характером силового воздействия на природные и искусственные массивы и их устойчивостью. В обоих случаях наблюдается линейный рост напряжений с увеличением времени, причем в обоих случаях зависимость является линейной (рис. 2).

Такая корреляция в поведении рудовмещающих массивов имеет практическую значимость для обоснования возможности использования затвердевших кольматированных хвостов подземного блокового выщелачивания руд, как альтернативы экономически неприемлемых бетонных твердеющих смесей. 

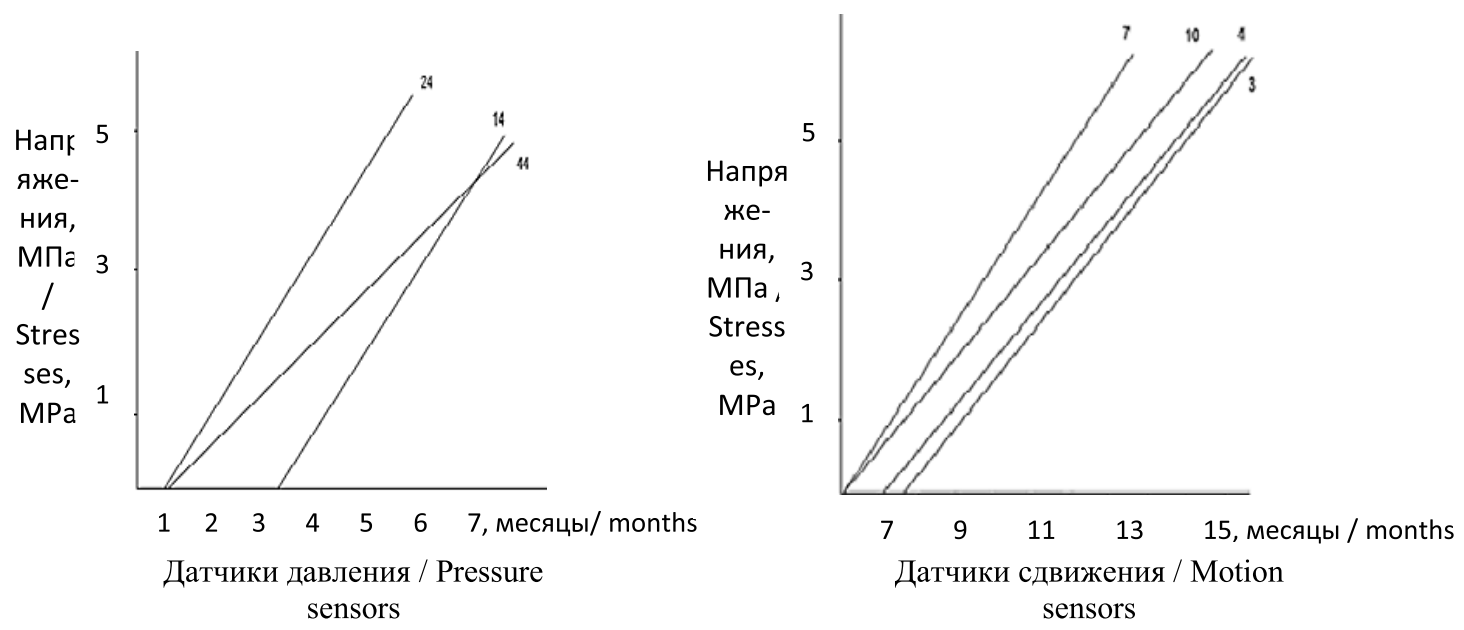

Рис. 2. Зависимость напряжений от времени /

Fig. 2. Dependence of the stress on time

Моделирование напряженности массива методом конечных элементов показывает, что на контуре выработок радиальные напряжения приближаются к 0, а тангенциальные - имеют максимальные значения.

Уменьшить время оценки напряженности породных кернов позволяет метод Голика-Денисова с нагружением образцов ступенями и полной разгрузкой на каждой ступени до появления остаточных деформаций. О напряженности массива судят по соотношению упругой и остаточной деформации.

Коэффициент удароопасности $K$ :

$$
K=\left(E_{\mathcal{M}}-E_{o}\right) / E_{o}=E_{y} / E_{o},
$$

где $E_{y}$ - упругая деформация, МПа;

$E_{o}$ - остаточная деформация, МПа;

$E_{м}$ - максимально достигнутая деформация, МПа;

Характер и закономерности распределения горного давления на жесткую металлическую арочную крепь устанавливают анализом показателей системы датчиков (рис. 3).

В прочных скальных породах в окрестностях горных выработок формируется зона пластических деформаций и разрушения пород, которая определяет поведение природных и искусственных массивов.

Излучатель акустических сигналов и звукоприемник помещали в скважинах глубиной 2,5 м, пробуренных на расстоянии 5 м друг от друга. Частота посылаемого в скважину сигнала составляла 2 кгц.

При прозвучивании породного массива размером 20x40м, сложенного алевролитами с коэффициентом структурного ослабления 0,2 , прочностью на сжатие 30-90 МПа, и на растяжение 3-3,5 МПа, сигнал перемещался со скоростью 3500 м/с.

При исследовании другого массива размерами 100х115 м в известняках с коэффициентом структурного ослабления 0,3 , пределом прочности на сжатие - 150 МПа и на растяжение 8-9 МПа скорость перемещения сигнала изменялась от 4000 до $6100 \mathrm{M} / \mathrm{c}$.

Объединение сравнимых значений коэффициента звукопоглощения изолиниями графически позволяет определить местоположение звукопоглощающего объекта на исследуемом участке месторождения (рис. 4) 


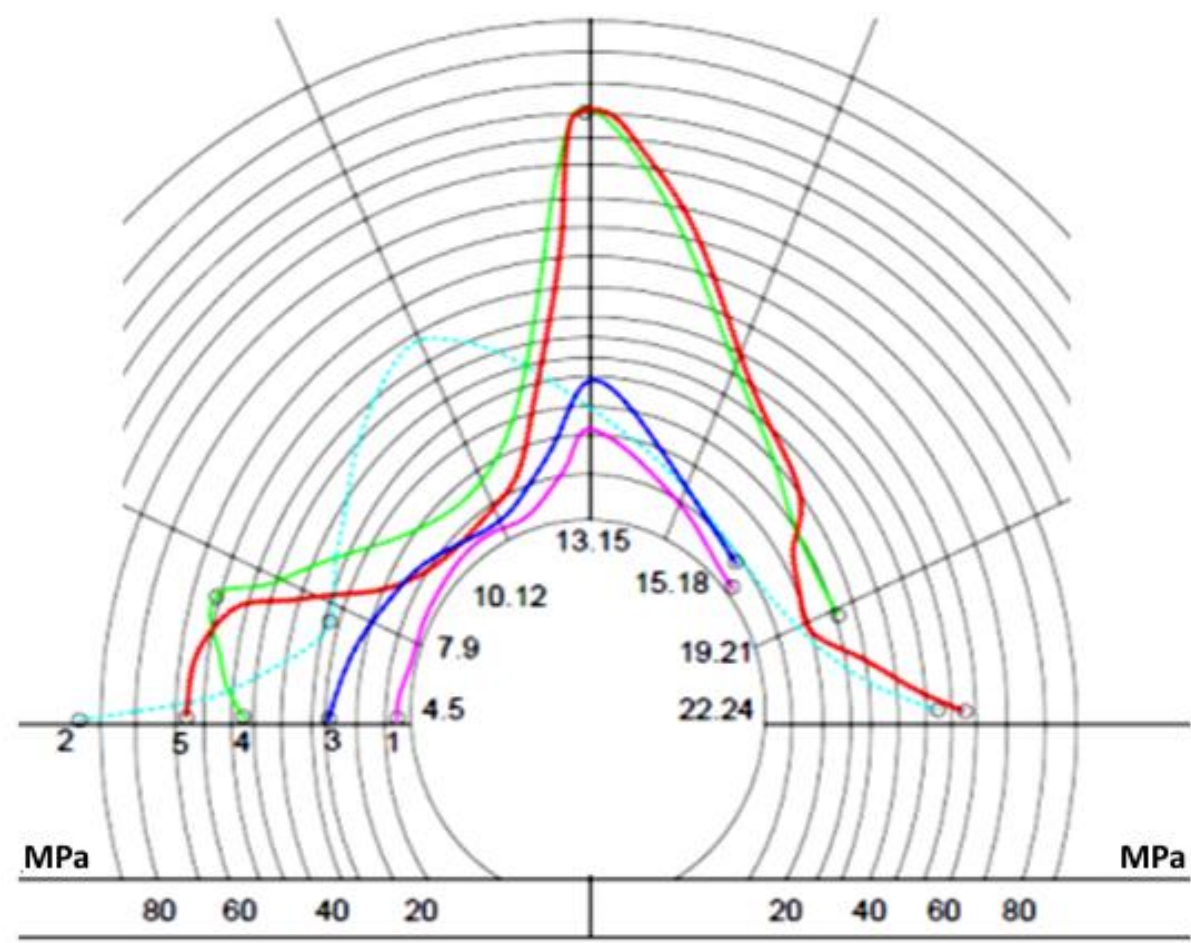

Рис. 3. Эпюры напряжений в металлической крепи: 4-24-тензометрические датчики; 1-5-замеры /

Fig. 3. Diagrams of stresses in the metal support: 4-24-strain-gage indicators; 1-5-measurements

Скв. 1 / Well 1

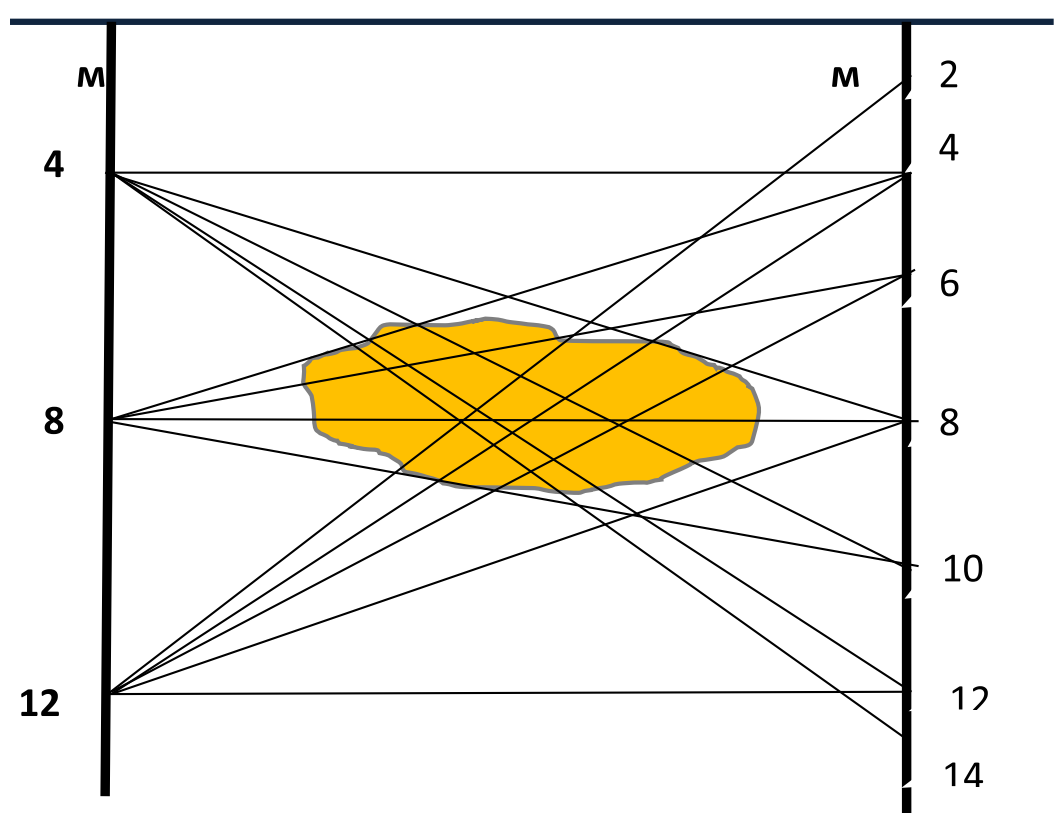

Рис. 4. Определение грании зоны неоднородности массива /

Fig. 4. Determination of the boundaries of the massif heterogeneity zone

Cкв. 2 / Well 2 


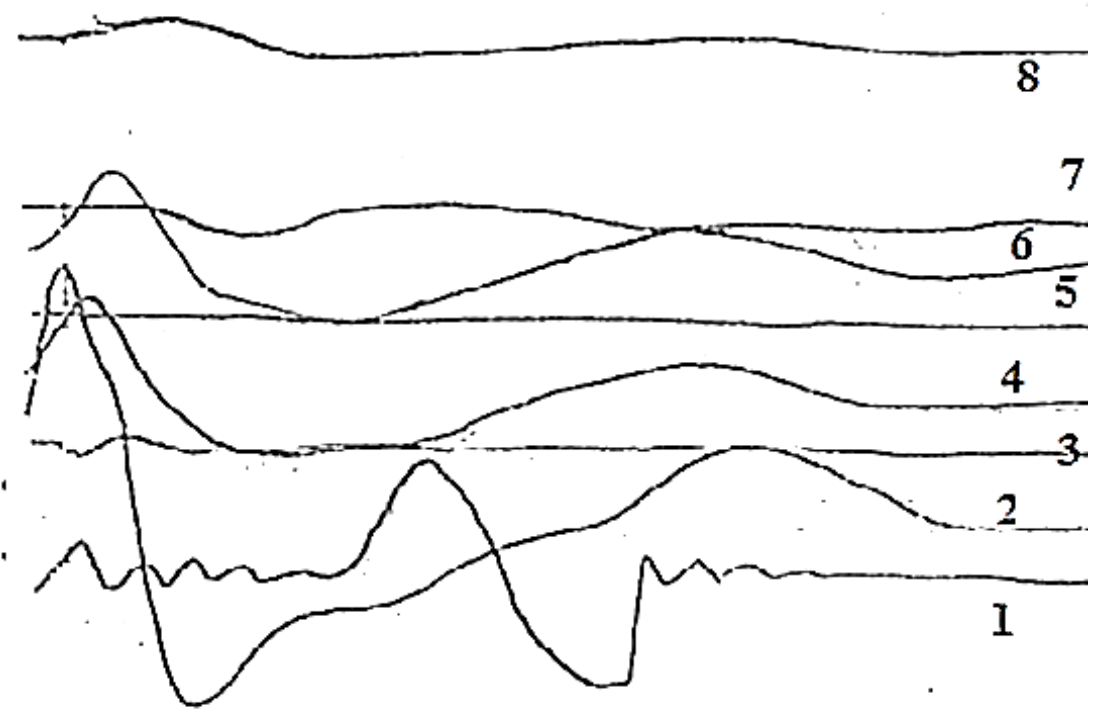

Рис. 5. Осииллограмма взрыва скважинь::1-8-датчики / Fig. 5. Oscillogram of the well blast: 1-8-sensors

Параметры фильтрационной неоднородности руд, определяющей возможность применения технологий, например, выщелачивания металлов реагентами, определяли по скорости распространения волн напряжений.

Упругие колебания создавали путем взрывания зарядов взрывчатых веществ весом 2 кг. Волны напряжений улавливали с помощью датчиков, устанавливаемых в 10 точках с соблюдением условия: один датчик - вертикально, а второй - горизонтально (рис. 5).

При осознании и интерпретации совокупного воздействия динамических и статических напряжений искусственный целик идентифицируется в виде заполненной закладочным материалом выработки радиусом $\mathbf{r}$ (рис. 6).

Возможность эффективного использования технологий обеспечивается средствами горной геофизики с получением ожидаемых результатов.

Заложенная камера /

Заложенная камера /

A filled chamber

A filled chamber

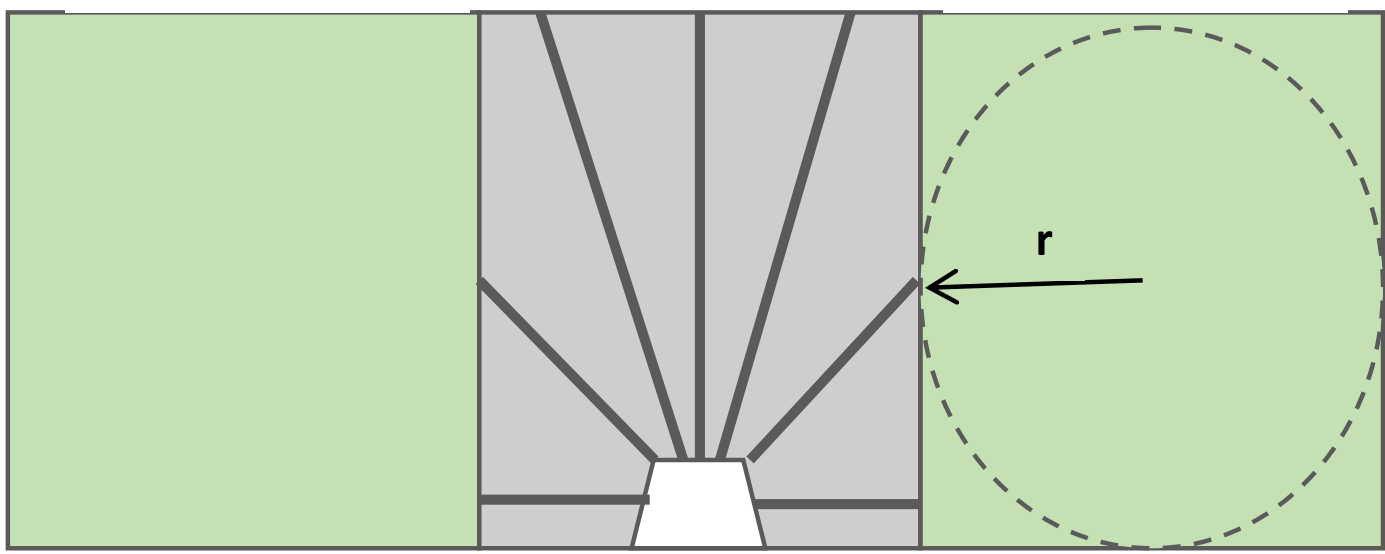

Рис. 6. Схема к определению нормальных и касательных напряжений /

Fig. 6. Scheme for determining normal and shear stresses 
Анализ теории и практики управления состоянием массивов при разработке скальных месторождений подземным способом показывает:

- область применения технологий с обрушением пород сокращается по экологическим условиям;

- способ погашения с оставлением выработанного пространства открытым применяется в редких случаях при хорошей изученности объекта;

- расширение диапазона технологий с закладкой твердеющими смесями ограничивается дефицитом компонент смесей, что определяет необходимость вовлечения в сферу производства отходов производства.

Концепция экономически экологически корректных технологий подземной разработки рудных месторождений включает в себя положения:

- геомеханическая сбалансированность дискретных сред рудовмещающих массивов является непременным условием эффективности управления ими с сохранением земной поверхности;

- технологии управления природными и искусственными массивами должны использовать феномен остаточной несущей способности структурных отдельностей путем перевода геоматериалов в режим объемного сжатия;

- рациональное использование свойств массивов достигается при комбинировании традиционных и новых способов добычи разносортных руд на основе феномена заполнения выработанного пространства выщелоченной рудой или твердеющими смесями.

Экономическая эффективность комбинирования технологий на основе геомеханики массивов описывается моделью:

$$
\Pi_{\kappa}=\left[\frac{3_{m p}}{A_{m p}} K_{\partial}^{A} K_{\partial}^{T}+\left(\frac{\ni_{m p}+\ni_{\xi}}{A_{m p}}\right)-\frac{3_{\sigma}}{A_{\sigma}}\right] A_{\kappa} \cdot r \ni=\left[\frac{3 \delta}{A \delta} K_{R}^{A} K_{P}^{T}+\left(\frac{\Delta \ni_{\ni}+\Delta \ni_{K}}{A_{\Phi}}\right)-\frac{3_{\Phi}}{A_{\Phi}}\right] A \cdot r
$$

где $\Pi_{\kappa}-$ прибыль от комбинирования технологий; $3_{m p}$ - затраты на единицу металла при базовой технологии, ден. ед.; $3_{6}$ - затраты на единицу металла при комбинированной технологии, ден. ед.; $K_{\partial}^{A}-$ коэффициент динамичности объемов производства; $K_{\partial}^{T}$ - коэффициент динамичности времени; э ${ }_{m p}-$ приведенные эксплуатационные и капитальные расходы при базовой технологии, ден. ед.; э. - приведенные эксплуатационные и капитальные расходы при комбинированной технологии, ден. ед.; $A_{m p}$ - годовой объем выпуска металлов при базовой технологии, ед.; $A_{m p}$ - годовой объем выпуска металлов при комбинированной технологии, ед.; $A_{\kappa}$ - годовой объем выпуска металлов по комбинированной технологии, ед.; $\mathrm{r}$ - коэффициент риска рыночных операций.

Снижение качества добытой базовой технологией руды за счет разубоживания увеличивает потери металлов на металлургическом заводе на 1-2\%. Использование комбинированных технологий повышает коэффициент извлечения на 1-1,5\%.

По данным анализа комбинирование технологий по сравнению с традиционной технологией уменьшает потери металлов при добыче на 5-10\%, а при обогащении на 2-2,5\%.

Технологии комбинируемых по критериям надежности и стоимости управления состоянием массива способствуют восстановлению промышленного потенциала Садонских месторождений [Дмитрак и др., 2006, 2019; Гавришев и др., 2017]. Совершенствование способов управления геомеханикой рудовмещающих массивов 
сопровождается модернизацией остальных процессов горного производства [Комащенко, 2016; Хулелидзе, 2016; Grikorkina et al., 2017; Zaalishvili et al., 2014 a, b, 2016, 2018; Milyukov et al., 2014; Горбатиков и др., 2015]. Результаты исследования согласуются с выводами исследователей по данному направлению горного производства [Feng, 2017; Jianju et al., 2017; Li, 2017; Zaalishvili et al., 2018].

\section{ВывоАЫ}

Поведение скальных массивов сложного строения при подземной разработке месторождений определяется совместным влиянием тектонических нарушений и остаточной несущей способности дискретных пород.

Сохранность дневной поверхности обеспечивается оптимальным соотношением напряжений в элементах геомеханической системы, которое контролируется геофизическими методами в рамках непрерывного мониторинга состояния горного массива и подземных инженерных коммуникаций.

Достоверность мониторинга состояния массива определяется комплексностью и возможностями используемых методов диагностики, включающих использование средств и методов геофизики.

\section{Аитература}

1. Гавришев С.Е., Корнилов С.Н., Пыталев И.А., Гапонова И.В. Повышение экономической эффективности горнодобывающих предприятий за счет вовлечения в эксплуатацию техногенных георесурсов. // Горный журнал. - 2017. - № 12. - С. 46-51.

2. Голик В.И., Бурдзиева О.Г., Дзеранов Б.В. Управление геомеханикой массива путем оптимизации технологии разработки. // Геология и геофизика Юга России. - 2020. - Т. 10. № 1. - С. 127-137. DOI: 10.23671/VNC. 2020.1.59070.

3. Голик В.И., Бурдзиева О.Г., Дзеранов Б.В., Чотчаев Х.О. Управление геодинамикой массива путем регулирования величины напряжений. // Геология и геофизика Юга России. -2020 . - Т. 10. №2. - С. 147-160. DOI: 10.46698/VNC. 2020.93.21.011

4. Дребенштедт К., Голик В.И., Дмитрак Ю.В. Перспективы диверсификации технологии добычи металлов в РСО-Алания. // Устойчивое развитие горных территорий. - 2018. - T. 10. № 1 (35). - C. 125-131.

5. Дмитрак Ю.В., Логачева В.М., Подколзин А.А. Геофизическое прогнозирование нарушенности и обводненности массива горных пород. // Горный информационно-аналитический бюллетень. - 2006. - № 11. - С. 35-36.

6. Дмитрак Ю.В., Цидаев Б.С., Дзапаров В.Х., Харебов Г.Х. Минерально-сырьевая база цветной металлургии России. // Вектор ГеоНаук. - 2019. - Т. 2. № 1. - С. 9-18.

7. Качурин Н. М., Стась Г.В., Корчагина Т.В., Змеев М.В. Геомеханические и аэрогазодинамические последствия подработки территорий горных отводов шахт Восточного Донбасса. // Известия Тульского государственного университета. Сер. Науки о Земле. 2017. - Вып. 1. - С. 170-182.

8. Клюев Р.В., Босиков И.И., Майер А.В., Гаврина О. А. Комплексный анализ применения эффективных технологий для повышения устойчивого развития природно-технической системы. // Устойчивое развитие горных территорий. - 2020. - №2. - С. 283-290.

9. Комащенко В.И. Разработка взрывной технологии, снижающей вредное воздействие на окружающую среду. // Известия Тульского государственного университета. Науки о Земле. - 2016. - № 1. - С. 34-43.

10. Рыльникова М.В., Владимиров Д.Я., Пыталев И. А., Попова Т. М. Роботизированные геотехнологии как путь повышения эффективности и экологизации освоения недр. // Физико-технические проблемы разработки полезных ископаемых. - 2017. - № 1. C. $92-101$. 
11. Хулелидзе К. К., Кондратьев Ю. И., Заалишвили В. Б., Бетрозов З. С. Оценка коренных и техногенных месторождений РСО-Алания как возможных объектов применения технологии подземного и кучного выщелачивания. // Устойчивое развитие горных территорий. - 2016. - T. 8. № 1. - C. 46-51.

12. Aksenov V.V., Khoreshok A.A., Beglyakov V.U., Efremenkov A.B. The concept of creating perspective technological paradigm of formation (development) of the underground space on the basis of the leading development of new approaches in construction geotechnology and geotechnics. Premises and basic provisions (part 1). // ISPCIET 2019. IOP Conf. Series: Materials Science and Engineering 656 (2019) 012004. IOP Publishing. - 2019.

13. Burdzieva O. G., Zaalishvili V. B., Beriev O. G., Kanukov A. S., Maysuradze M. V. Mining impact on environment on the North Ossetian territory. // International Journal of GEOMATE. 2016. - Vol. 10 (1). - pp. 1693-1697.

14. Feng X.-T. Rock mechanics and engineering. Vol. 4: Excavation, Support and Monitoring. $1^{\text {st }}$ edition. CRC Press. // Taylor\&Francis Group. - 2017. - 726 p.

15. Grigorkina G.S., Ramonova A.G., Kibizov D.D., Kozyrev E.N., Zaalishvili V.B., Magkoev T.T., Fukutani K. Probing specific oxides as potential supports for metal/oxide model catalysts: MgO (111) polar film. // Solid State Communications. - 2017. - Vol. 257. - pp. 16-19.

16. Jianju Du, Xiang Huiqin, Qingli Zeng, Luqing Zhang, Qunce Chen, Jian Zhou, Wen Meng. Estimation of the present-day stress field using in-situ stress measurements in the Alxa area, Inner Mongolia for China's HLW disposal. // Engineering Geology. - 2017. - Vol. 220. - pp. 76-84.

17. Li N.N., Chai S.B., Li H. B. Analytical study of ground motion caused by seismic wave propagation across faulted rock masses. // International Journal for Numerical and Analytical Methods in Geomechanics. - 2017. - Vol. 42. No. 1. - pp. 95-109.

18. Milyukov V.K., Yushkin V.D., Kopaev A.V., Mironov A.P., Demyanov G.V., Sermyagin R.A., Basmanov A. V., Popadev V. V., Nasretdinov I. F., Zaalishvili V. B., Kanukov A. S., Dzeranov B. V. Monitoring current vertical movements of the Northern Caucasus by absolute and relative gravimetry. // Measurement Techniques. - 2014. - Vol. 56. No. 10. - pp. 1105-1110.

19. Zaalishvili V.B., Melkov D.A. Reconstructing the Kolka surge on September 20, 2002 from the instrumental seismic data. // Izvestiya. Physics of the Solid Earth. - 2014. - Vol. 50. No. 5. - pp. 707-718.

20. Zaalishvili V.B., Nevskaya N. I., Melkov D.A. Instrumental geophysical monitoring in the territory of Northern Caucasus. // Izvestiya. Physics of the Solid Earth. - 2014. - Vol. 50. No. 2. - pp. 263-272.

21. Zaalishvili V. B., KanukovA. S., Melkov D. A., Makiev V.D., Dzobelova L. V.Development of a unified model of geoinformation system for city planning and integration.// International Journal of GEOMATE. - 2018. - Vol. 15. No. 51. - pp. 160-166.

\section{References}

1. Gavrishev S.E., Kornilov S.N., Pytalev I.A., Gaponova I.V. Increasing the economic efficiency of mining enterprises due to the involvement of anthropogenic geo-resources in the operation. Mining journal. 2017. No. 12. pp. 46-51. (In Russ.)

2. Golik V.I., Burdzieva O. G., Dzeranov B. V. Management of rock mass geomechanics by optimizing development technology. Geology and Geophysics of Russian South. 2020. Vol. 10. No. 1. pp. 127-137. DOI: 10.23671/VNC. 2020.1.59070 (In Russ.)

3. Golik V. I., Burdzieva O. G., Dzeranov B. V., Chotchaev Kh. O. Controlling the geodynamics of the massif by regulating the level of stresses. Geology and Geophysics of Russian South. 2020. Vol. 10. No. 2. pp. 147-160. DOI: 10.46698/VNC. 2020.93.21.011 (In Russ.)

4. Drebenstedt K., Golik V.I., Dmitrak Yu. V. Prospects for diversification of metal mining technology in North Ossetia-Alania. Sustainable development of mountain territories. 2018. Vol. 10. No. 1 (35). pp. 125-131. (In Russ.)

5. Dmitrak Yu. V., Logacheva V.M., Podkolzin A.A. Geophysical forecasting of disturbance and watering of the rock mass. Mining information and analytical bulletin. 2006. No. 11. pp. 35-36. (In Russ.) 
6. Dmitrak Yu. V., Tsidaev B.S., Dzaparov V.Kh., Kharebov G. Kh. Mineral resource base of non-ferrous metallurgy in Russia. Vector GeoSciences. 2019. Vol. 2. No. 1. pp. 9-18. (In Russ.)

7. Kachurin N.M., Stas G.V., Korchagina T.V., Zmeev M.V. Geomechanical and aerogasdynamic consequences of undermining the territories of allotments of mines in the Eastern Donbass. Bulletin of the Tula State University. Ser. Earth Sciences. 2017. Issue 1. pp. 170-182. (In Russ.)

8. Klyuev R. V., Bosikov I. I., Mayer A. V., Gavrina O.A. Comprehensive analysis of the use of effective technologies to improve the sustainable development of the natural and technical system. Sustainable development of mountain territories. 2020. No. 2. pp. 283-290. (In Russ.)

9. Komashchenko V.I. Development of an explosive technology that reduces the harmful effect on the environment. Bulletin of the Tula State University. Earth Sciences. 2016. No. 1. pp. 34-43. (In Russ.)

10. Rylnikova M. V., Vladimirov D. Ya., Pytalev I.A., Popova T. M. Robotic geotechnology as a way to increase efficiency and greening of subsoil development. Physical and technical problems of the mineral development. 2017. No. 1. pp. 92-101. (In Russ.)

11. Khulelidze K.K., Kondratyev Yu. I., Zaalishvili V.B., Betrozov Z.S. Assessment of primary and technogenic deposits in North Ossetia-Alania as possible objects of application of underground and heap leaching technology. Sustainable development of mountain territories. 2016. Vol. 8. No. 1. pp. 46-51. (In Russ.)

12. Aksenov V.V., Khoreshok A.A., Beglyakov V.U., Efremenkov A.B. The concept of creating perspective technological paradigm of formation (development) of the underground space on the basis of the leading development of new approaches in construction geotechnology and geotechnics. Premises and basic provisions (part 1). ISPCIET 2019. IOP Conf. Series: Materials Science and Engineering 656 (2019) 012004.

13. Burdzieva O. G., Zaalishvili V. B., Beriev O. G., Kanukov A. S., Maysuradze M. V. Mining impact on environment on the North Ossetian territory. International Journal of GEOMATE. 2016. Vol. 10 (1). pp. 1693-1697.

14. Feng X.-T. Rock mechanics and engineering. Vol. 4, Excavation, Support and Monitoring. $1^{\text {st }}$ edition. CRC Press. Taylor\&Francis Group. 2017. 726 p.

15. Grigorkina G.S., Ramonova A. G., Kibizov D.D., Kozyrev E.N., Zaalishvili V.B., Magkoev T.T., Fukutani K. Probing specific oxides as potential supports for metal/oxide model catalysts: $\mathrm{MgO}$ (111) polar film. Solid State Communications. 2017. Vol. 257. pp. 16-19.

16. Jianju Du, Xiang Huiqin, Qingli Zeng, Luqing Zhang, Qunce Chen, Jian Zhou, Wen Meng. Estimation of the present-day stress field using in-situ stress measurements in the Alxa area, Inner Mongolia for China's HLW disposal. Engineering Geology. 2017. Vol. 220. pp. 76-84.

17. Li N. N., Chai S.B., Li H.B. Analytical study of ground motion caused by seismic wave propagation across faulted rock masses. International Journal for Numerical and Analytical Methods in Geomechanics. 2017. Vol. 42. No. 1. pp. 95-109.

18. Milyukov V.K., Yushkin V.D., Kopaev A.V., Mironov A.P., Demyanov G.V., Sermyagin R.A., Basmanov A. V., Popadev V. V., Nasretdinov I. F., Zaalishvili V. B., Kanukov A. S., Dzeranov B. V. Monitoring current vertical movements of the Northern Caucasus by absolute and relative gravimetry. Measurement Techniques. 2014. Vol. 56. No. 10. pp. 1105-1110.

19. Zaalishvili V.B., Melkov D.A. Reconstructing the Kolka surge on September 20, 2002 from the instrumental seismic data. Izvestiya. Physics of theSolidEarth. 2014. Vol. 50. No. 5. pp. 707-718.

20. Zaalishvili V.B., Nevskaya N.I., Melkov D.A. Instrumental geophysical monitoring in the territory of Northern Caucasus. Izvestiya. Physics of the Solid Earth. 2014. Vol. 50. No. 2. pp. 263-272.

21. Zaalishvili V. B., KanukovA. S., Melkov D. A., Makiev V.D., Dzobelova L. V. Development of a unified model of geoinformation system for city planning and integration. International Journal of GEOMATE. 2018. Vol. 15. No. 51. pp. 160-166 